\title{
Exploration of economical 3D printer
}

\author{
Mingchao $\mathrm{Yu}^{1, \mathrm{a}^{*}}$ and Chong $\mathrm{Tan}^{2, \mathrm{~b}}$ \\ ${ }^{1}$ Key Laboratory of Metallurgical Equipment and Control Technology, Ministry of Education, \\ Wuhan University of Science and Technology, Wuhan 430081, China. \\ ${ }^{2}$ Hubei Key Laboratory of Mechanical Transmission and Manufacturing Engineering, \\ Wuhan University of Science and Technology, Wuhan 430081, China. \\ a503616860@qq.com, ${ }^{b}$ tc18983100080@163.com
}

Keywords: 3D printer; Desktop; Cantilever; Rapid prototyping; Slice processing.

\begin{abstract}
In view of the diversification of the demand of many users for 3D printing technology and the high price of $3 \mathrm{D}$ printers in the current market, it hinders the widespread application and promotion of 3D printers and seriously affects the development of 3D printing technology, and now proposed a home-based, universal, and based on three-freedom cantilever mechanical structure of the $3 \mathrm{D}$ printer exploration program. This 3D printer in the structural improvements expand the scope of the print, and the software design makes the user interface more friendly and humane, and the combination of the two also makes the machine slightly smaller size, lower costs. In general, it can meet the large most pay-for-home or individual, business needs for cost-effective.
\end{abstract}

\section{Introduction}

In recent years, 3D printing technology, as a rapid prototyping system based on a three-dimensional digital software model, is gradually influencing and changing the traditional processing and manufacturing industry. Such as biomedicine, architecture, vehicles, archaeology and other fields. However, due to the relatively high cost of producing 3D printers domestically, just an ordinary 3D printer can cost tens of thousands of RMB each, which has a certain financial burden to the average small and medium-sized enterprise or a family that is quite interested in this. To a certain extent, this restricts about the promotion and dissemination of 3D printing technology among the private sector as well as the public sector hinders its technological development. In view of this, based on the family desktop 3D printing technology, using a three-degree-of-freedom cantilever mechanical structure combined with desktop 3D printing layering technology to design an economical 3D printer for home use. To fill the market for low-cost and have the corresponding printing requirements of the 3D printer market gaps to meet the general public and SMEs urgently needed it.

\section{The Development of Family 3D Printer}

At present, 3D printing technology is gradually breaking through the traditional machining manufacturing industry, becoming the hot manufacturing technology of the emerging manufacturing industry at this stage. Its rapid processing and molding can meet the actual needs of most enterprises or individuals and has certain promotional value. Moreover, applying its rapid prototyping technology to our daily life will surely bring great convenience. Using science and technology in service is also the ultimate goal of our country to vigorously develop science and technology. Sometimes, the use of home-desktop 3D printer can quickly and easily create the objects we need. If the processing material to choose the right, machined objects in all aspects of performance can also meet the requirements of users, and experiencing this process of processing can also be for the operator and even children at home to bring the use fun of science and technology. However, the price of existing home-based 3D printers is barely affordable for the majority of middle-income families, and more families in the middle and lower incomes are expected to purchase a 3D printer for various actual needs or purposes. Therefore, proper structural 
improvements to existing desktop 3D printers on the market should reduce their production costs to meet the economic needs that most households and even individuals can buy at will [1].

The domestic and foreign research on 3D printers are not the same. Many foreign printer version is open source, and in its network developer provides a full set of free source code, mechanical structure drawings and circuit control schematics, the algorithm is also faster replacement, operating efficiency is relatively high, to meet its Real needs of their own nationals. In view of this, the domestic development of family desktop 3D printer is also growing. So in recent years there are also emerged in various versions of open source 3D printers. However, when compared with each other, most of the domestic enterprises only simplified the source code and the computer interface abroad, lacking their own core technical support or innovative changes. In general, the development of the domestic 3D printer is still a little bit disproportionate to that of other countries. This requires not only continuous research by many researchers but also vigorous support from relevant departments. Therefore, the above problems aims to extract several special features in different printers and software systems to do the creative mix and improve in order to achieve a more family-oriented, low-cost purpose [2].

\section{D Printing Technology Principle and Structure Design}

At present, 3D printer design has two types of open and closed structure. Among them, a structural model that is earlier in the market is open-type. Through the in-depth research of 3D printing technology in recent years, the open-structured 3D printers are gradually withdrawn from the market by the scientific researchers. The closed-structure 3D printers are gradually emerging. A revolution in 3D printing technology is also getting hotter and hotter [3].

Working principle. Many people are aware that the rapid prototyping technology itself is based on the virtual digital model file, the use of low melting point of hot-melt materials and rapid prototyping features through the material extrusion mechanism in the terminal nozzle heating and extrusion of the extruder, extruding the printed material in a very fine filament form and then "printed" layer by layer according to a previously programmed print path at the computer system interface [4]. After a certain period of accumulation, the desired product is finally obtained.

The entire 3D printer system consists of PC and 3D printer two parts. Among them, the host computer's role is mainly two: model design and the three-dimensional slices of model. In other words, 3D printers are usually printed on a specific path by layer according to pre-designed control instructions on a computer to accomplish the desired work [5]. Among them, the pre-designed 3D model is the basis for 3D printing. Under normal circumstances, usually using 2D or 3D software AutoCAD, SolidWorks, etc. designed and stored as STL or SLA and other file formats. At the same time, after designing the 3D model that you need, you also need to check it. If it is found that the model size is not a special match with the printer size, you need to make appropriate changes to the model size in order to meet the requirements. Data processing diagram shown in Figure 2.

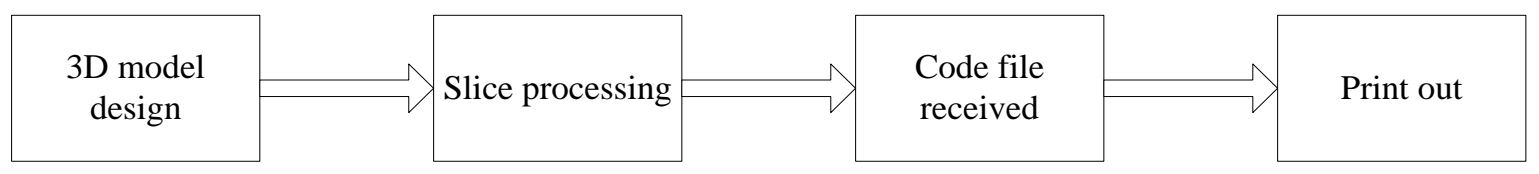

Figure $13 \mathrm{D}$ printing data processing block diagram

In order to achieve the rapid prototyping effect that a 3D printer should have, first of all, the appropriate slice software is used to slice the model before printing begins. After the completion of the slicing work, you need to use this slicing software to translate the information about the shape, size and other information of the sliced object into the $\mathrm{G}$ codes that the $3 \mathrm{D}$ printer can recognize to facilitate the normal printing operation of the printer [6].

Mechanical structure. There are two types of mechanical structures currently available in the market. They are gantry type and cantilever type, respectively. As the executive mechanism of the $3 \mathrm{D}$ printer, taking into account the respective advantages and disadvantages and economy of the two types of structures, and aiming at the problem that the printing platform of gantry type 3D 
printer is fixed and the printing space is limited, so here select cantilever mechanical structure design. Cantilever 3D mechanical structure consists of four parts, including: rotary motion mechanism, vertical motion mechanism, horizontal motion mechanism, material extrusion mechanism, and each part comes with a separate servo motor drive [7]. The material extrusion mechanism is mounted on the print head of the 3D printer and utilizes the molten material to be fed and withdrawn by the intermeshing action of the molten material between the rollers and the servomotor gears in the interior of the extrusion mechanism. Due to the special movement mode of the cantilever mechanical structure, that is, the material forming and printing relies mainly on the vertical rotational movement and the horizontal linear movement, which is less horizontal than the traditional gantry mechanical structure motion guide, so it will not be limited by the print space, print a larger range.

The specific mechanism is as follows: the print head on the extruding mechanism is connected with the horizontal guide rail slider by the fixing part on the print head, while the horizontal guide rail slider is connected with the horizontal guide rail by the ball screw nut pair, and driven by a servo motor so that the print head can be moved horizontally [8]. The combination of these devices constitutes the cantilever device of the mechanical structure of the cantilever type 3D printer, and at the same time, satisfies the radius parameter in the cylindrical coordinate system. Similarly, the above-mentioned cantilever assembly is mechanically connected to the vertical guide rail by means of a connecting plate and driven by a single vertical servo motor to move the entire boom assembly up and down, this constitutes the vertical coordinate in the cylindrical coordinate system. Then, the vertical guide is connected by the supporter and the base rotation unit of the mechanism, and the vertical guide is driven by the other separate bottom rotary axis servo motor to make a rotary motion together with the boom device, thereby establishing the arc parameter of the cylindrical coordinate system. At this point, the entire 3D printer integrated the above three mechanical structures to establish a rotating motion, horizontal movement and up and down movement of three degrees of freedom mechanical structure [9].

\section{Control System Design}

Control system belongs to the brain-level status for any organization, so the control system as the core of the 3D printer is also equivalent to its "brain". After the computer receives the optimized G code instruction designed in advance from the slicing software, Followed by a comprehensive analysis of the computer will be carried out by the computer and then set the path control servo motor in accordance with the set path to move, and finally the expected print tasks to complete the finished print [10]. Control system hardware block diagram shown in Figure 2.

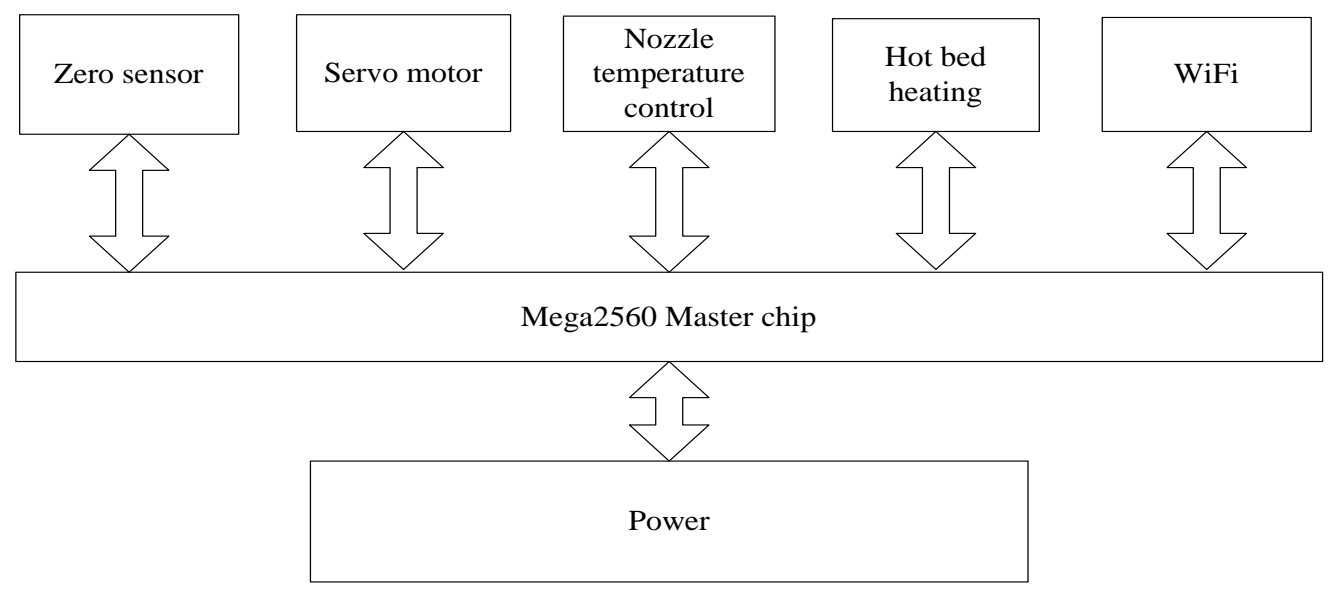

Figure 2 hardware block diagram of 3D printer control system

Here the control system uses mega2560 as the master chip, in which the zero sensor is installed in the $\mathrm{X}, \mathrm{Y}, \mathrm{Z}$ axis end, used to initialize the coordinate system to determine the maximum motor movement; Servo motor drive module is driven printer nozzle according to the computer to set a 
good path for movement; WiFi module is the focus of wireless control data transmission, so that it can achieve long-range wireless control; Nozzle temperature control module mainly controls the voltage across the single-head heater in order to be able to indirectly control the nozzle of the temperature, to achieve the function of adjusting the temperature, the nozzle temperature stabilized at the expected setting, so that the printing material can quickly melt forming. Finally, the hot bed heating module is heated by the heating circuit so that the bed has a certain temperature so that printed material can be fully attached to the hot bed for easy printing, in general with the nozzle temperature control module is very similar [11].

\section{Interactive Interface Design}

For desktop economy 3D printer, in order to make it family, it must meet the following conditions: 1) With affordable income in the family can accept the price; 2) The specifications cannot be too large, buy back the whole machine can be placed on a common home table; 3) The software itself also has a higher stability, so as not to affect the user experience, in general, is the ability to print in place $[12]$; 4) The last one is easy to use, that is for a novice user in a certain period of time to understand and learn "fool" print operation [13].

Taking into account the home-made desktop 3D printer from the model to the completion of the finished product to complete the steps, and the combination of the above four conditions for this desktop-based home-based low-cost 3D printer control software interface design is as follows: For a home-based 3D printer, the human-computer interface must be "friendly", and the main interface of the software by the title bar, text menu, shortcut menu, the main view area, the overall information display area, the summary information display area six small areas, and with a title bar at the top to display information such as the file name and a text menu next to the title bar [14]. This menu provides the most basic operational options for most software user interfaces, including: New, Open, Save, Layer, Fill, View, Model, G code, and then arranged in order and easy to operate; And in the main view area, the mouse wheel can be used to achieve real-time scaling of the interface and the model of arbitrary rotation; Shortcut menu bar, as the name suggests is to provide users with familiar with the flow of operations to achieve the role of rapid operation; Right The profile information is placed symmetrically to the shortcut menu bar, with summary information including main view, top view, right view switching, and model information such as model size, number of patches, layer thickness, number of layers, fill spacing and number of paths, And this column is also provided to the user more intuitive understanding of the various parameters of the model place; The user interface provides the user with almost the vast majority of useful information for the user, the interface of each ribbon may not necessarily be used to, but at least provided to the user the possibility of selection, to pick for their own valuable information [15].

\section{Conclusions}

Aiming at the structural design problem of home economical 3D printer, this paper innovatively combines the three degrees of freedom cantilever mechanical structure with the control software and system of desktop 3D printer to design a 3D printer that is suitable for most family. With the progress of society and the progress of science and technology, the new technology industry of 3D printing will get hotter and hotter, and it more and more will bring convenience to the common people. The market of 3D printing will also expand, so we cannot make high-cost constraints become a constraint on the development of 3D printing technology. And taking this into consideration, it is imperative to have a certain amount of structural optimization of existing home-based 3D printers. At the same time, due to the growing maturity of 3D printing technology, users have been enabled to directly use the Internet to operate the printer remotely, and the items that can be printed are also more and more diverse, more and more able to meet the needs of various fields, Therefore, based on the above situations, the innovation of 3D printing technology will get more and more attention from all walks of life. Finally, based on the flaws existing in finished 
products printed by many existing 3D printers, whether we can finally repair and improve them with some systems or software is worth our continued research and discussion.

\section{References}

[1] Z.Q. Zhang, Z.L. Wang, Y.W. Chen, Z.X. Pang, L. Wang and C.C. Ma. Journal of Machine Design, Vol. 33 (2016) No.11, p.56.

[2] S.L. Han, Z.Y. Li, Y. Xiao and X. Xu. Machinery Design \& Manufacture, (2015) No.11, p.116.

[3] P. Chen, Y. Liu and Y.L. Mei. Journal of Machine Design, Vol. 34 (2017) No.2, p.126.

[4] W. Cheng, X.Y. Li, J.P. Dai and M.M. Cheng. Machinery, Vol. 29 (2016) No.4, p.97.

[5] Q. Ge and Y. Wang. Modern Electronics Technique, Vol.39 (2016) No.22, p.100.

[6] G.F. Deng. Mechanical Engineer, (2014) No.12, p.134.

[7] K. Zheng. Technology Innovation and Application, (2017) No.10, p.92.

[8] Z.H. Wang, Y.B. Xue and L.N. Xu. Techniques of Automation and Applications, Vol. 36 (2017) No.3, p.130.

[9] Q. Xia, B. Zhou and Z.F. Lin. Techniques of Automation and Applications, Vol. 36 (2017) No.6, p.158.

[10] H.T. Zhao, X. Dong, G. Wu and N. Xing. Techniques of Automation and Applications, Vol. 35 (2016) No.9, p.122.

[11]F. Guan, T.C. Ge and L. Liu. Mechatronics, Vol. 22 (2016) No.4, p.53.

[12] M.J. Wang, H.C. Wei, M.X. Xiao and X. Wei. Modern Electronics Technique, Vol. 40 (2017) No.12, p. 108.

[13]B. Ran and G.C. Zhang. SHEJI, (2015) No.14, p.145.

[14] X.L. Li, J.X. Ma and P. Li. Process Automation Instrumentation, Vol. 35 (2014) No.1, p.1.

[15]D.J. Zhou. Electro-Mechanical Engineering, Vol. 33 (2017) No.2, p.13. 